\section{Cladism defended}

THE contribution of L.B. Halstead to your journal was so misinformed about the assumptions and benefits of phylogenetic analysis that we are compelled to comment. A thorough presentation of phylogenetics ('cladistics') is inappropriate, but we do wish to make some remarks.

Phylogenetic methods are used to determine the most parsimonious arrangement of the patterns of character distribution among taxa (groups of organisms). These patterns are assumed to be the results of evolutionary processes and to reflect natural, monophyletic groups. The result of the analysis is a cladogram, a branching diagram with each branch justified by a character thought to be a unique evolutionary innovation. It should be noted that these diagrams are in fact hypotheses about relative recency of common ancestry among the taxa considered. 'Relationship' is thus defined by a genealogical criterion rather than such vague concepts as proportion of shared genes, as evidenced by supposed morphological similarity, or estimation of overall morphological similarity.

Dr Halstead's remarks about the phylogenetic system being designed such that fossils cannot be included is simply uninformed: many phylogeneticists have dealt with this issue, including one of us (E.O.W.) who published a phylogenetic analysis of fossil and Recent gar fishes.

Dr Halstead seems to be most interested in those evolutionary changes that 'allow' organisms to enter new 'adaptive zones', concepts widely used by grade-oriented systematists. He states, "the fact that many parallel changes can be related to adaptations to similar conditions, is more significant than listing trivial trademarks from which cladograms can be constructed." But, with some reflection, that statement yields a simple fact: parallel changes in evolution are not discernable, indeed are incomprehensible, without a genealogical hypothesis of relationship. Put simply, how can we know that similarity shared between two organisms is a parallelism without a phylogenetic tree which demonstrates that the character was not, in fact, found in the common ancestral species of the two taxa? This concept is so basic that we find it difficult to understand the misconceptions concerning it. Further, the "trivial trademarks" which mark monophyletic groups are often the very characters which are associated with new "adaptive zones" (for example, feathers of birds and mammary glands of mammals).

Dr Halstead states that if one could trace the ancestry of man back in time to a rhipidistian fish, the whole sequence of changes would, by definition, be a single monophyletic species. He adds that "most zoologists and palaeontologists" would surely use arbitrary divisions to partition that lineage. This misses another basic biological fact. The lineage is broken up, from the Devonian to the Recent. If this were not so, we would observe only one tetrapod species, Homo sapiens, and not the diversity of land vertebrates that we observe today. (Wiley (Syst. Zool., 11-21, 1978) has shown that conclusions such as Halstead's stem from an artificial concept of classification.)

Since "the issue was summed up for many by R. Parrington's exasperated exclamation that according to the cladists a lungfish is more closely related to a cow than to a salmon," it is appropriate to end with a comment on that statement. It is not the cladist who 'makes' a lungfish more closely related to a cow than to a salmon any more than it is a cladist who 'makes' Hyracotherium more closely related to Equus than to Eocene condylarths. Evolutionary history as exemplified by genealogical descent with modification (a Darwinian concept) 'makes' it so. Lungfishes and cows are more closely related to each other than lungfishes are to salmons because lungfishes and cows share a common ancestor that is not shared with salmons. That this is somehow 'wrong' indicates a confusion on the part of critics about the meaning of genealogical relationship. If cladograms, with their clearly defined data and clearly stated hypotheses, "are difficult enough for experts in the field to comprehend fully," then we suggest that the shortcomings lie with the 'experts' and not with phylogenetic methods or their results.

Museum of Comparative Zoology

William L. Fink

Harvard University

Cambridge, Massachusetts 02138

E.O. WILEY

Museum of Natural History and the

Department of Systematics and Ecology,

University of Kansas,

Lawrence, Kansas 66045

As a non-participant in the scientific meeting of which Dr Halstead's report has stirred such sharp reactions in your columns, but as a previous exponent (Classification and Biology, Heinemann, 1970) of the principles excoriated by Halstead under the name of 'cladism', I would like to address four questions to him. First, would he agree that there is not, and probably cannot be, an objective measure of 'degree of similarity' between organisms in respect of phenotypic characters of the type studied by museum systematists, and in particular that the 'unit character' of the numerical taxonomists has proved to be undefinable? Second, would he accept that the most fundamental type of similarity or difference between organisms is that of their genotypes, as represented primarily by their nuclear DNA? Third, would he accept that we now have objective methods of measuring DNA similarity, either directly (by DNA hybridisation and similar techniques) or indirectly (by comparative protein studies) and that the range and reliability of data from these techniques is likely to increase? Fourth, if by such methods it could be shown that a lungfish was 'nearer' to a cow than to a salmon, would $\mathrm{Dr}$ Halstead then be prepared to accept the 'cladist' case in this instance. And if the answer to this question were "yes", would this alter his general attitude to phylogenetic classification in the sense proposed by Hennig (and myself)?

The University,

R. A. Crowson

Glasgow W2

HALSTEAD states that the phylogenetic ideas illustrated in the "....cladograms presented by $P$. Janvier (University of Paris) on agnathans and B. Gardiner (Queen Elizabeth College, London) on choanichthyans ... were demolished within hours of their being proposed"'. I wish to reply to this statement, which is designed to dismiss cladistics in general and Dr Gardiner and myself in particular.
I proposed the following phylogeny based on cladistic methodology.

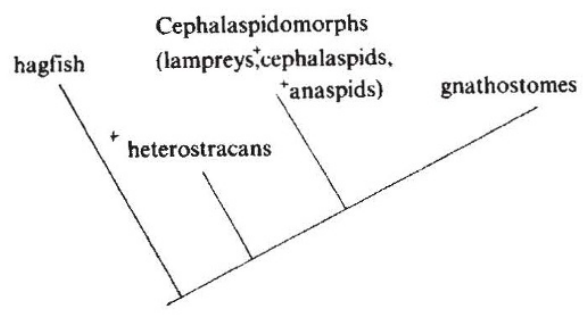

An objection was raised by Dr Halstead who did not agree with the proposed affinities of the Heterostraci, a group of poorly known fossil agnathans. He pointed out that heterostracans probably had paired nostrils and nasal sacs (diplorhiny) and were therefore related to gnathostomes and not to other agnathans which are monorhinous. This information is supposed to demolish my phylogeny and to support his own 'evolutionary tree' given below (with permission):

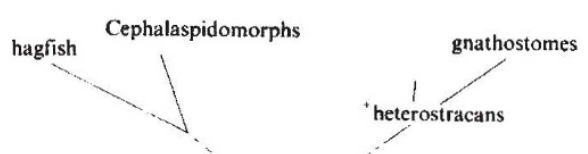
We agree that the diplorhinal condition is a
primitive craniate character-state. Therefore, Halstead must believe that primitiveness is a criterion by which we recognise relationship. As a cladist I cannot accept this view.

If heterostracans did have paired nostrils (and not all palaeontologists accept this interpretation) then I have to recognise that monorhiny arose twice; in the hagfish and in cephalaspidomorphs. To counter this objection I could lean on detailed embryological and anatomical information to argue that the hagfish monorhiny had arisen independently of that seen in the lamprey. But this approach is both fruitless and unnecessary in the light of other information which I presented. Furthermore, basing one's case on whether heterostracans were monorhinal or diplorhinal is a particularly unfortunate tactic. Heterostracans are represented by thin bony shields and the interpretation of the soft anatomy is governed by the recent model we choose. This is amply demonstrated by the differing views of heterostracan morphology proposed by Stensio ${ }^{1}$ and Halstead 2 for instance.

Unless Halstead can provide derived character-states to show that heterostracans are more closely related to gnathostomes than to other agnathans then I submit that the 'demolition' amounts to no more than a dislike of my idea because it differs from his.

Laboratoire de Paléontologie

Philippe JANVIER

Université Paris VI

4, Place Jussieu

75230 Paris

1. Stensio, E.A. In Current Problems of Lower Vertebrate Phylogeny, Nobel Symposium 4, Almqvist \& Wiksell (1968). 2. Halstead, L.B. Biol. J. Linn. Soc. Lond. 5: 339-349 (1973). 